\title{
Holographic Schwinger Effect in a Confining D3-Brane Background with Chemical Potential
}

\author{
Zi-qiang Zhang, ${ }^{1}$ De-fu Hou, ${ }^{2}$ Yan Wu, ${ }^{1}$ and Gang Chen ${ }^{1}$ \\ ${ }^{1}$ School of Mathematical and Physics, China University of Geosciences (Wuhan), Wuhan 430074, China \\ ${ }^{2}$ Key Laboratory of Quark and Lepton Physics (MOE), Central China Normal University, Wuhan 430079, China \\ Correspondence should be addressed to Zi-qiang Zhang; zhangzq@cug.edu.cn
}

Received 7 June 2016; Revised 12 August 2016; Accepted 23 August 2016

Academic Editor: Elias C. Vagenas

Copyright (c) 2016 Zi-qiang Zhang et al. This is an open access article distributed under the Creative Commons Attribution License, which permits unrestricted use, distribution, and reproduction in any medium, provided the original work is properly cited. The publication of this article was funded by $\mathrm{SCOAP}^{3}$.

\begin{abstract}
Using the AdS/CFT correspondence, we investigate the Schwinger effect in a confining D3-brane background with chemical potential. The potential between a test particle pair on the D3-brane in an external electric field is obtained. The critical field $E_{c}$ in this case is calculated. Also, we apply numerical method to evaluate the production rate for various cases. The results imply that the presence of chemical potential tends to suppress the pair production effect.
\end{abstract}

\section{Introduction}

Schwinger effect is known as the pair production in an external electric field in QED [1]. The virtual electronposition pairs can become real particles when a strong electric field is applied. The production rate $\Gamma$ has been calculated in the weak-coupling and weak-field approximation long time ago. Later, it is generalized to arbitrary-coupling and weakfield case in [2]

$$
\Gamma \propto e^{-\pi m^{2} / e E+e^{2} / 4},
$$

where $m$ and $e$ are the mass and charge of the created particles, respectively, $E$ is the external electric field. This nonperturbative effect can be explained as a tunneling process and is not restricted to QED but usual for QFTs coupled to $U(1)$ gauge field.

AdS/CFT, namely, the duality between type IIB superstring theory formulated on $\mathrm{AdS}_{5} \times S^{5}$ and $\mathcal{N}=4$ SYM in four dimensions, can realize a system that coupled with $U(1)$ gauge field [3-5]. Therefore, it is very interesting to consider the Schwinger effect in a holographic setup. After the works $[6,7]$ in which the creation rate of the quark pair in $\mathcal{N}=4$ SYM theory was obtained firstly, there are many attempts to address Schwinger effect in this direction. For instance, the universal aspects of this effect in the general backgrounds are investigated in [8]. The pair production in confining background is studied in $[9,10]$. The potential barrier for the pair creation is analyzed in [11]. The pair production in the conductivity of a system of flavor and color branes is analyzed in [12]. The Schwinger effect with constant electric and magnetic fields is investigated in $[13,14]$. The nonrelativistic Schwinger effect is discussed in [15]. The Gauss-Bonnet corrections to this effect are studied in [16]. This effect is also studied with some AdS/QCD models $[17,18]$. Other important results can be found, for example, in [19-24]. For a recent review on this topic, see [25].

As we know, a finite temperature of the gauge theory is on the gravity side of the duality represented by a black hole horizon in the bulk located at $r_{t}$ in the fifth coordinate and extended in the other four space-time directions. In addition, a chemical potential of the gauge theory can be obtained in this setup by giving the black hole an electric charge. Since the Schwinger effect in a confining D3-brane background has been discussed in [9], it is interesting to consider the influence of the chemical potential on Schwinger effect in this case. In this paper, we will add the chemical potential to the confining D3-brane background. We would like to see how chemical potential affects the Schwinger effect. It is the motivation of the present work. 
The organization of this paper is as follows. In the next section, the background with chemical potential is briefly introduced. In Section 3, we perform the potential analysis in this background. Then we investigate the production rate in Section 4. The last part is devoted to conclusion and discussion.

\section{Background with Chemical Potential}

In the framework of the AdS/CFT duality, $\mathcal{N}=4$ SYM theory with a chemical potential is obtained by making the black hole in the holographic dimension be charged. The corresponding metric is an asymptotical $\mathrm{AdS}_{5}$ space with a Reissner-Nordstrom black hole. Its metric can be written as [26]

$$
\begin{aligned}
& d s^{2} \\
& =\frac{r^{2}}{L^{2}}\left[-\left(d x^{0}\right)^{2}+\left(d x^{1}\right)^{2}+\left(d x^{2}\right)^{2}+f(r)\left(d x^{3}\right)^{2}\right] \\
& +\frac{L^{2}}{r^{2}} f(r)^{-1} d r^{2}+L^{2} d \Omega_{5}^{2}
\end{aligned}
$$

with

$$
f(r)=1-\left(1+Q^{2}\right)\left(\frac{r_{t}}{r}\right)^{4}+Q^{2}\left(\frac{r_{t}}{r}\right)^{6},
$$

where $L$ is the AdS space radius, the string tension $1 / 2 \pi \alpha^{\prime}$ relates the 't Hooft coupling constant by $L^{2} / \alpha^{\prime}=\sqrt{\lambda}$ and $r$ denotes the radial coordinate of the black brane geometry. $r_{t}$ is the inverse compactification radius in $x^{3}$-direction. As $r_{t}$ grows, the radius shrinks.

The chemical potential $\mu$ is given by

$$
\mu=\frac{\sqrt{3} Q r_{t}}{L^{2}}
$$

where the charge $Q$ of the black hole is in the range $0 \leq Q \leq$ $\sqrt{2}$.

Note that when $Q=0$ (zero chemical potential), the usual confining D3-brane background is reproduced. In addition, when $r_{t}=0$ (zero temperature), the usual $\mathrm{AdS}_{5} \times S^{5}$ background is reproduced.

We should point out that the chemical potential implemented in this way is not the quark (or baryon) chemical potential of QCD but a chemical potential that refers to the $R$-charge of $\mathcal{N}=4$ SYM theory. However, we can apply it as a simple way of introducing finite density effects into the system in the present work. More discussions about the AdS-RN in the context of holographic setups can be found in $[27,28]$.

\section{Potential Analysis}

3.1. Potential Research. We now perform the potential analysis for confining D3-brane background with chemical potential using the metric equation (2). The classical string action can reduce to the Nambu-Goto action

$$
S=T_{F} \int d^{2} \sigma \sqrt{g}=T_{F} \int d \tau d \sigma \mathscr{L}, \quad T_{F}=\frac{1}{2 \pi \alpha^{\prime}},
$$

where $g$ is the determinant of the induced metric on the string world sheet embedded in the target space; that is,

$$
g_{\alpha \beta}=g_{\mu \nu} \frac{\partial X^{\mu}}{\partial \sigma^{\alpha}} \frac{\partial X^{\nu}}{\partial \sigma^{\beta}},
$$

where $X^{\mu}$ and $g_{\mu \nu}$ are the target space coordinates and the metric and $\sigma^{\alpha}$ with $\alpha=0,1$ parameterize the world sheet.

By using the static gauge,

$$
\begin{aligned}
x^{0} & =\tau, \\
x^{1} & =\sigma, \\
r & =r(\sigma),
\end{aligned}
$$

we can obtain the induced metric $g_{\alpha \beta}$ :

$$
\begin{aligned}
g_{00}= & \frac{r^{2}}{L^{2}}, \\
g_{01}= & g_{10}=0, \\
g_{11} & \\
= & \frac{r^{2}}{L^{2}} \\
& +\frac{L^{2}}{r^{2}\left[1-\left(1+Q^{2}\right)\left(r_{t} / r\right)^{4}+Q^{2}\left(r_{t} / r\right)^{6}\right]}\left(\frac{\partial r}{\partial \sigma}\right)^{2} .
\end{aligned}
$$

Then the Lagrangian density becomes

$$
\mathscr{L}=\sqrt{\frac{1}{1-\left(1+Q^{2}\right)\left(r_{t} / r\right)^{4}+Q^{2}\left(r_{t} / r\right)^{6}} \dot{r}^{2}+\frac{r^{4}}{L^{4}}},
$$

with $\dot{r}=d r / d \sigma$.

Now that $\mathscr{L}$ does not depend on $\sigma$ explicitly, we have the conserved quantity,

$$
\mathscr{L}-\frac{\partial \mathscr{L}}{\partial \dot{r}} \dot{r} .
$$

The boundary condition at $\sigma=0$ is

$$
\begin{aligned}
& \dot{r}=0, \\
& r=r_{c}
\end{aligned}
$$

$$
\left(r_{t}<r_{c}<r_{0}\right)
$$

which leads to

$$
\begin{aligned}
& \frac{r^{4}}{\sqrt{\left(1 /\left(1-\left(1+Q^{2}\right)\left(r_{t} / r\right)^{4}+Q^{2}\left(r_{t} / r\right)^{6}\right)\right) \dot{r}^{2}+r^{4} / L^{4}}} \\
& \quad=C=r_{c}^{2} L^{2} ;
\end{aligned}
$$

then a differential equation is derived,

$$
\begin{aligned}
\dot{r} & =\frac{d r}{d \sigma} \\
& =\frac{r^{2} \sqrt{r^{4}-r_{c}^{4}}}{r_{c}^{2} L^{2}} \sqrt{1-\left(1+Q^{2}\right)\left(\frac{r_{t}}{r}\right)^{4}+Q^{2}\left(\frac{r_{t}}{r}\right)^{6}} .
\end{aligned}
$$


By integrating (13) the separate length $x$ of the test particle pair on the D3-brane becomes

$$
\begin{aligned}
x= & \frac{2 L^{2}}{r_{0} a} \\
& \cdot \int_{1}^{1 / a} \frac{d y}{y^{2} \sqrt{\left(y^{4}-1\right)\left[1-\left(1+Q^{2}\right)(b / a y)^{4}+Q^{2}(b / a y)^{6}\right]}},
\end{aligned}
$$

where the following dimensionless parameters have been introduced:

$$
\begin{aligned}
& y \equiv \frac{r}{r_{c}}, \\
& a \equiv \frac{r_{c}}{r_{0}}, \\
& b \equiv \frac{r_{t}}{r_{0}} .
\end{aligned}
$$

Plugging (13) into (9), one obtains the classical action. Then the sum of potential energy (PE) and static energy (SE) of string is obtained as

$$
\begin{aligned}
& V_{\mathrm{PE}+\mathrm{SE}} \\
& =2 T_{F} r_{0} a \int_{1}^{1 / a} \frac{y^{2} d y}{\sqrt{\left(y^{4}-1\right)\left[1-\left(1+Q^{2}\right)(b / a y)^{4}+Q^{2}(b / a y)^{6}\right]}} .
\end{aligned}
$$

3.2. The Critical Field. To ensure that the potential analysis is right and consistent with the DBI result, we should pause here to gain the critical field by DBI action at hand.

The DBI action is

$$
S_{\mathrm{DBI}}=-T_{\mathrm{D} 3} \int d^{4} x \sqrt{-\operatorname{det}\left(G_{\mu \nu}+\mathscr{F}_{\mu \nu}\right)},
$$

where $T_{\mathrm{D} 3}$ is the $\mathrm{D} 3$-brane tension:

$$
T_{\mathrm{D} 3}=\frac{1}{g_{s}(2 \pi)^{3} \alpha^{\prime 2}} \text {. }
$$

In terms of the metric equation (2), the induced metric $G_{\mu \nu}$ reads

$$
\begin{aligned}
G_{00} & =-\frac{r^{2}}{L^{2}}, \\
G_{11} & =\frac{r^{2}}{L^{2}}, \\
G_{22} & =\frac{r^{2}}{L^{2}}, \\
G_{33} & =\frac{r^{2}}{L^{2}}\left[1-\left(1+Q^{2}\right)\left(\frac{r_{t}}{r}\right)^{4}+Q^{2}\left(\frac{r_{t}}{r}\right)^{6}\right] \\
& =\frac{r^{2}}{L^{2}} f(r) .
\end{aligned}
$$

After considering $\mathscr{F}_{\mu \nu}$ term, which can be written as $\mathscr{F}_{\mu \nu}=2 \pi \alpha^{\prime} F_{\mu \nu}[29]$, we find

$$
\begin{aligned}
G_{\mu \nu}+\mathscr{F}_{\mu \nu} & \left(\begin{array}{cccc}
-\frac{r^{2}}{L^{2}} & 2 \pi \alpha^{\prime} E_{1} & 2 \pi \alpha^{\prime} E_{2} & 2 \pi \alpha^{\prime} E_{3} \\
-2 \pi \alpha^{\prime} E_{1} & \frac{r^{2}}{L^{2}} & 0 & 0 \\
-2 \pi \alpha^{\prime} E_{2} & 0 & \frac{r^{2}}{L^{2}} & 0 \\
-2 \pi \alpha^{\prime} E_{3} & 0 & 0 & \frac{r^{2}}{L^{2}} f(r)
\end{array}\right),
\end{aligned}
$$

which leads to

$$
\begin{aligned}
\operatorname{det}\left(G_{\mu \nu}+\mathscr{F}_{\mu \nu}\right) \\
=-\frac{r^{4}}{L^{4}} f(r)\left[\frac{r^{4}}{L^{4}}-\left(2 \pi \alpha^{\prime}\right)^{2}\left(E_{1}^{2}+E_{2}^{2}+\frac{E_{3}^{2}}{f(r)}\right)\right] .
\end{aligned}
$$

Here the electric field is only turned on $x^{1}$-direction [9]; one can take $E_{2}=E_{3}=0$ in (21), which yields

$$
\operatorname{det}\left(G_{\mu \nu}+\mathscr{F}_{\mu \nu}\right)=-\frac{r^{4}}{L^{4}} f(r)\left[\frac{r^{4}}{L^{4}}-\left(2 \pi \alpha^{\prime}\right)^{2} E^{2}\right],
$$

where we have replaced $E_{1}$ by $E$ for simplification.

Plugging (22) into (17) and making the D3-brane be located at $r=r_{0}$, we have

$$
S_{\mathrm{DBI}}=-T_{\mathrm{D} 3} \frac{r_{0}^{4}}{L^{4}} \int d^{4} x \sqrt{f(r)\left[1-\frac{\left(2 \pi \alpha^{\prime}\right)^{2} L^{4}}{r_{0}^{4}} E^{2}\right]} .
$$

To avoid the action equation (23) being ill-defined, it is required that

$$
1-\frac{\left(2 \pi \alpha^{\prime}\right)^{2} L^{4}}{r_{0}^{4}} E^{2} \geq 0
$$

where we have used the assumption

$$
f(r) \geq 0 .
$$

So the range of electric field is

$$
E \leq \frac{1}{2 \pi \alpha^{\prime}} \frac{r_{0}^{2}}{L^{2}}
$$

Finally, the critical field $E_{c}$ in confining D3-brane background with chemical potential is obtained; that is,

$$
E_{c}=\frac{1}{2 \pi \alpha^{\prime}} \frac{r_{0}^{2}}{L^{2}} .
$$

Note that $E_{c}$ is not affected by the chemical potential. In other words, the critical field $E_{c}$ in this case is consistent with that in confining D3-brane background. 


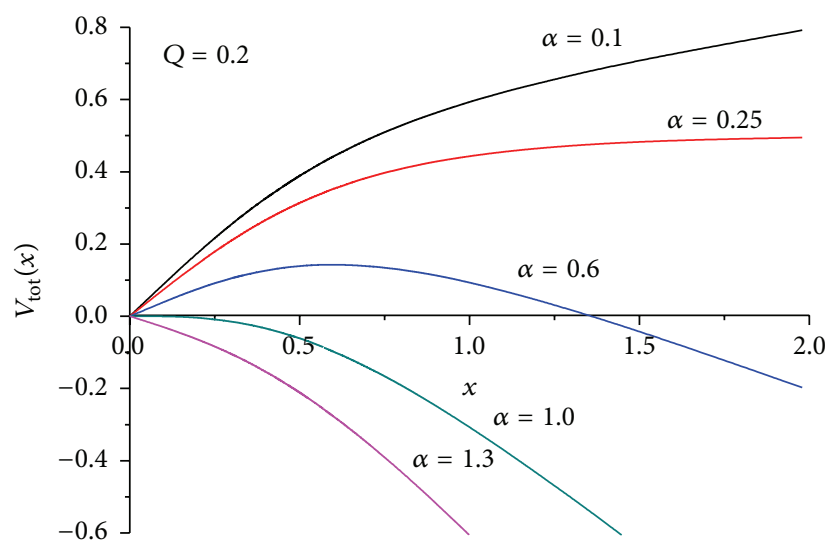

(a)

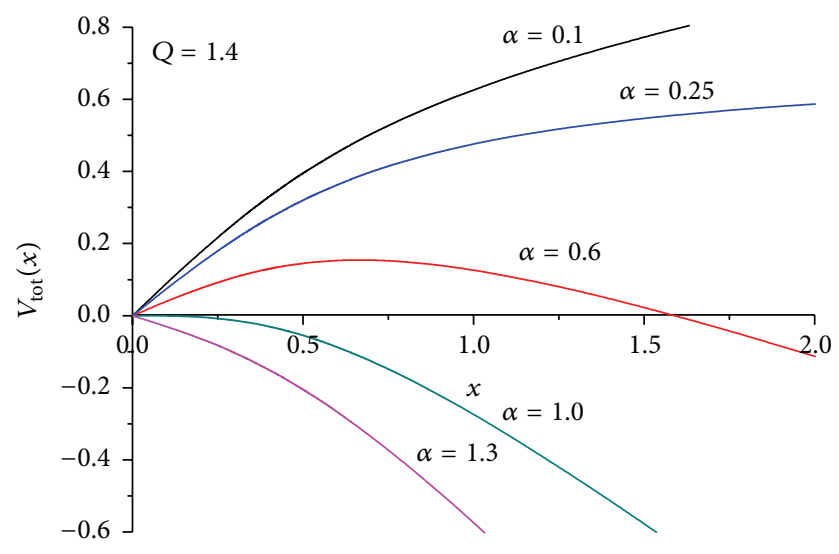

(b)

Figure 1: The total potential versus distance $x$ with two different values of $Q$. (a) $Q=0.2$; (b) $Q=1.4$. In all of the plots from top to bottom $\alpha=0.1,0.25,0.6,1.0,1.3$.

3.3. Total Potential. To proceed, we compute the total potential. For convenience, we introduce a dimensionless parameter

$$
\alpha \equiv \frac{E}{E_{c}} .
$$

Then, from (14) and (16), the total potential $V_{\text {tot }}$ can be written as

$$
\begin{aligned}
V_{\text {tot }} & =V_{\mathrm{PE}+\mathrm{SE}}-E x \\
& =2 T_{F} r_{0} a \int_{1}^{1 / a} \frac{y^{2} d y}{\sqrt{\left(y^{4}-1\right)\left[1-\left(1+Q^{2}\right)(b / a y)^{4}+Q^{2}(b / a y)^{6}\right]}} \\
& -\frac{2 T_{F} \alpha r_{0}}{a} \\
& \cdot \int_{1}^{1 / a} \frac{d y}{y^{2} \sqrt{\left(y^{4}-1\right)\left[1-\left(1+Q^{2}\right)(b / a y)^{4}+Q^{2}(b / a y)^{6}\right]}} .
\end{aligned}
$$

We have checked that the total potential $V_{\text {tot }}$ in confining D3-brane background can be derived from (29) if we neglect the effect of chemical potential by plugging $Q=0$ in (29).

Before discussing the results, let us recall the potential analysis in confining D3-brane background. There exist two critical values of the electric field, $E_{s}=T_{F} r_{t}^{2} / L^{2}$ and $E_{c}=$ $T_{F} r_{0}^{2} / L^{2}$. When $E<E_{s}$, the pair is confined and no Schwinger effect can occur. When $E_{s}<E<E_{c}$, the potential barrier is present and the pair production is described as a tunneling process. As $E$ increases, the potential barrier decreases gradually. At last, it vanishes at $E=E_{c}$. When $E>E_{c}$, no tunneling occurs and the production rate is not exponentially suppressed any more, the pair production is catastrophic, and the vacuum becomes totally unstable.

We now discuss the results. The total potential can be plotted versus the separate distance of the test particle pair on the D3-brane numerically. Here Figure 1 is plotted for a fixed value of $b$ (fixed temperature) and $Q$ (related to chemical potential) with different values of $\alpha$. To compare with the case in [9], we take $b=0.5$ and $2 L^{2} / r_{0}=r_{0} /\left(\pi \alpha^{\prime}\right)=1$ here. In
Figure 1, Figure 1(a) is plotted for a small value of chemical potential $(Q=0.2)$ while Figure $1(b)$ is for a larger value of chemical potential $(Q=1.4)$. In all of the plots from top to bottom $\alpha=0.1,0.25,0.6,1.0,1.3$. From the figures, we can indeed see that there exist two critical values of the electric field: one is related to $\alpha=1\left(E=E_{c}\right)$ and the other is at $\alpha=0.25\left(E=E_{s}=0.25 E_{c}\right)$.

In order to see the effect of chemical potential clearly, we plot the potential versus $x$ with fixed $\alpha$ and varied $Q$ in Figure 2. From Figure 2(a), we can see that as $Q$ increases the height and width of the potential barrier both increase. Meanwhile, from Figure 2(b), we can see that as $Q$ increases the height of the potential that becomes flat also increases. As we know, higher potential barrier makes the produced pair harder to escape to infinity. So the presence of chemical potential tends to suppress the Schwinger effect. In addition, as shown in Figure 2(b), one of the critical values of the electric field $\left(E=E_{s}=0.25 E_{c}\right)$ is not affected by the chemical potential. This can be also understood by looking at the value of $E_{s}$ defined as $E_{s}=T_{F} r_{t}^{2} / L^{2}$.

\section{Production Rate}

In this section, we study the pair production rate. Here we consider an AdS soliton background [10]

$$
\begin{gathered}
d s^{2}=\frac{L^{2}}{z^{2}}\left[-\left(d x^{0}\right)^{2}+\left(d x^{1}\right)^{2}+\left(d x^{2}\right)^{2}\right. \\
\left.+f(z)\left(d x^{3}\right)^{2}+\frac{d z^{2}}{f(z)}\right]+L^{2} d \Omega_{5}^{2},
\end{gathered}
$$

with

$$
f(z)=1-\left(1+Q^{2}\right)\left(\frac{z}{z_{t}}\right)^{4}+Q^{2}\left(\frac{z}{z_{t}}\right)^{6},
$$

where $x^{3}$-direction is compactified on a circle $S^{1}$ with radius $R=\pi z_{t}$. The AdS boundary is at $z=0$; the geometry is cut off at $z=z_{t}$. 


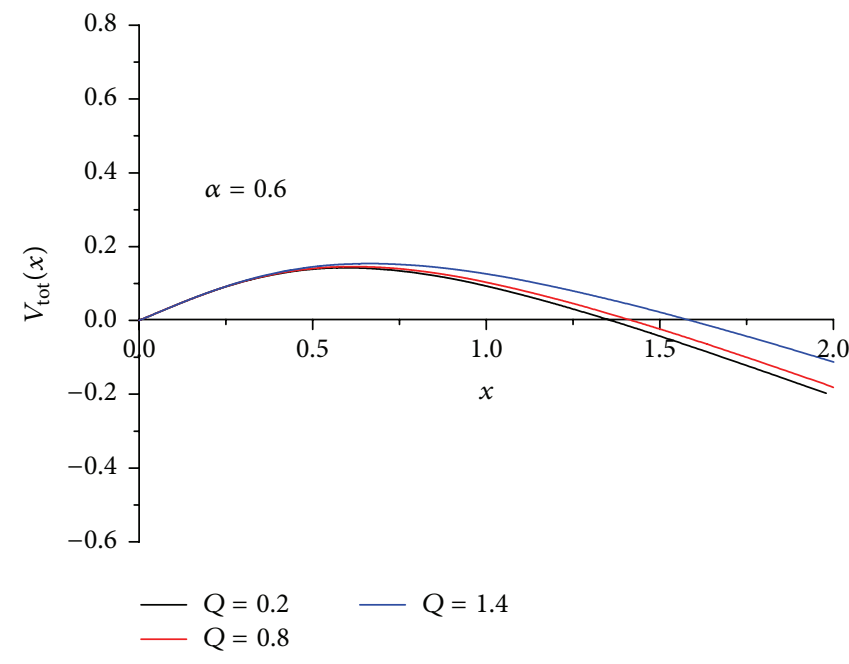

(a)

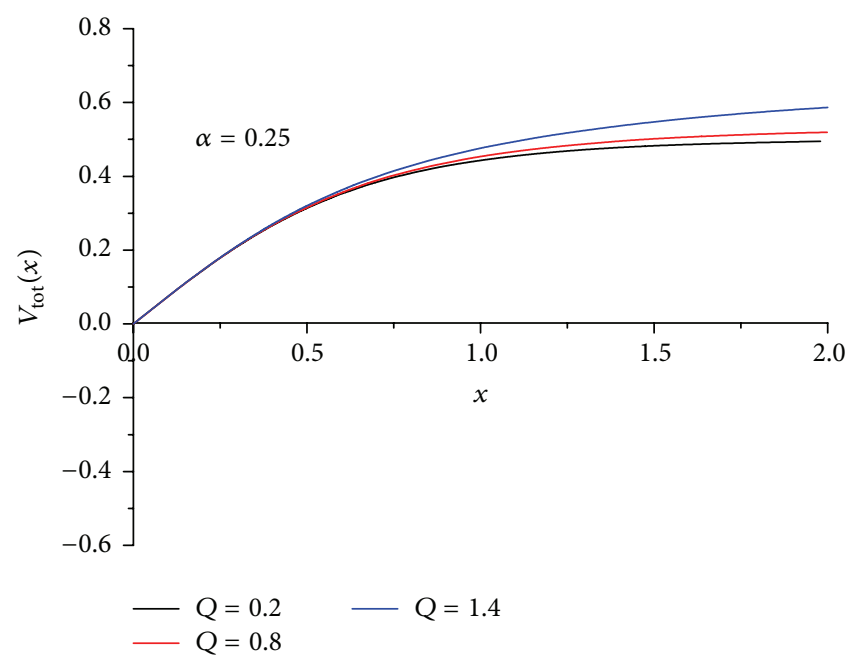

(b)

Figure 2: The total potential versus distance $x$ with fixed $\alpha$ and varied $Q$. (a) $\alpha=0.6$; (b) $\alpha=0.25$. In all of the plots from top to bottom $Q=0.2,0.8,1.4$.

The holographic pair production rate is [7]

$$
\Gamma \sim e^{-\left(S_{\mathrm{NG}}+S_{B_{2}}\right)} .
$$

To evaluate the pair production rate, one needs to compute the action or the expectation value of a circular Wilson loop on the probe brane. In [10], the circular Wilson loop is chosen to lie in $\tau$ - $\sigma$ plane. As a matter of convenience, here we use the polar coordinate, $(r, \theta)$, as follows from [16], so the string worldsheet can be parameterized by $z=z(r)$ in this case. With this ansatz, the induced metric is given by

$$
d s^{2}=\frac{L^{2}}{z^{2}}\left[\left(1+\frac{z^{\prime 2}}{f(z)}\right) d r^{2}+r^{2} d \theta^{2}\right],
$$

with $z^{\prime}=d z / d r$.

Then the string action can be expressed as

$$
\begin{gathered}
S_{\mathrm{NG}}=2 \pi L^{2} T_{F} \int_{0}^{x} d r \frac{r}{z^{2}} \sqrt{1+\frac{z^{\prime 2}}{f(z)}}, \\
S_{B_{2}}=-2 \pi T_{F} B_{01} \int_{0}^{x} d r r=-\pi E x^{2},
\end{gathered}
$$

where the external electric field is defined as $E \equiv T_{F} B_{01}$ with $B_{01}$ the nonvanishing component of $B_{2}$ and $x$ stands for the radius of the circular Wilson loop. By minimizing the action, one obtains the equation of motion

$$
z^{\prime \prime}(1+r)+\frac{2 r z^{\prime 2}}{z}-\frac{r z^{\prime 2}}{2 f(z)} \frac{d f(z)}{d z}+\frac{2 r f(z)}{z}=0
$$

The boundary conditions of (35) are [14]

$$
\begin{aligned}
z^{\prime}\left(z=z_{c}\right) & =0, \\
z(r=x) & =z_{0}, \\
z^{\prime}(r=x) & =-\left.\sqrt{f(z)\left(\frac{1}{\alpha^{2}}-1\right)}\right|_{z=z_{0}},
\end{aligned}
$$

where $\alpha$ is defined in (28).

To proceed further, we need to turn to numerical methods. To compare with the case in [10], we take $2 \pi L^{2} T_{F}=10$ and set the endpoint of the soliton as $z_{t}=1$. Then the whole action is only dependent on $\alpha, z_{0}$, and $Q$. In Figure 3, we plot the classical action $S$ and the exponential factor $e^{-S}$ against $\alpha$ with fixed $Q=0.2$. Other cases with different $Q$ have similar picture. As shown in these figures, the action diverges around a certain value which leads to a vanishing $e^{-S}$. We know from the above section that when $\alpha<\alpha_{s}$, with $\alpha_{s} \equiv E_{s} / E_{c}=z_{0}^{2} / z_{t}^{2}$, the Schwinger effect does not occur. Thus, this agreement supports that the numerical results of the production rate are consistent with the potential analysis.

To see the effect of the chemical potential on Schwinger effect, we plot $e^{-S}$ versus $\alpha$ at fixed $z_{0} / z_{t}$ with two different values of $Q$ in Figure 4 . From the plots, we can see that $e^{-S}$ decreases as $Q$ increases. This means that the chemical potential suppresses the pair production rate and also agrees with the previous potential analysis. Moreover, as $z_{0} / z_{t}$ increases, the effect of the chemical potential becomes weaker; this phenomenon can be found in Figure 4(b), where the two curves with different $Q$ are nearly overlapped.

\section{Conclusion and Discussion}

Schwinger effect in confining gauge theories may be an important ingredient in looking for new aspects of QCD in 


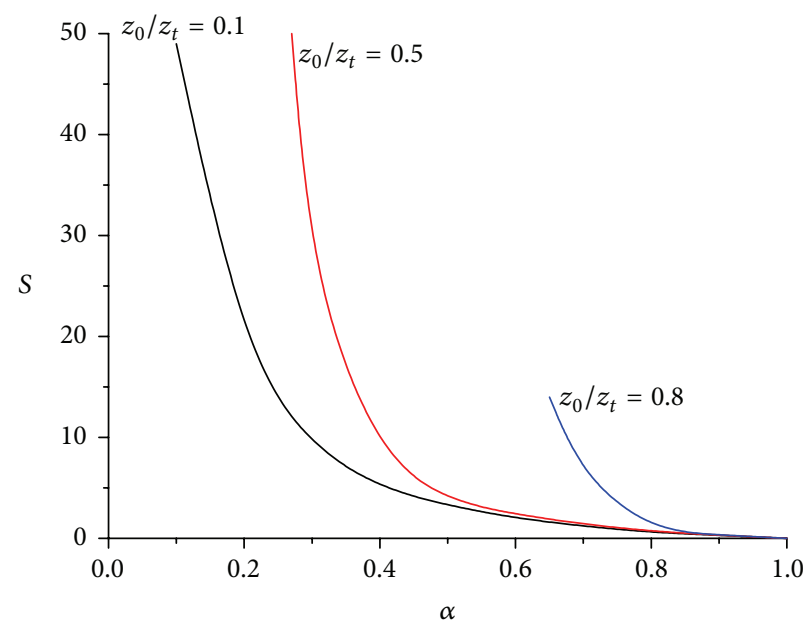

(a)

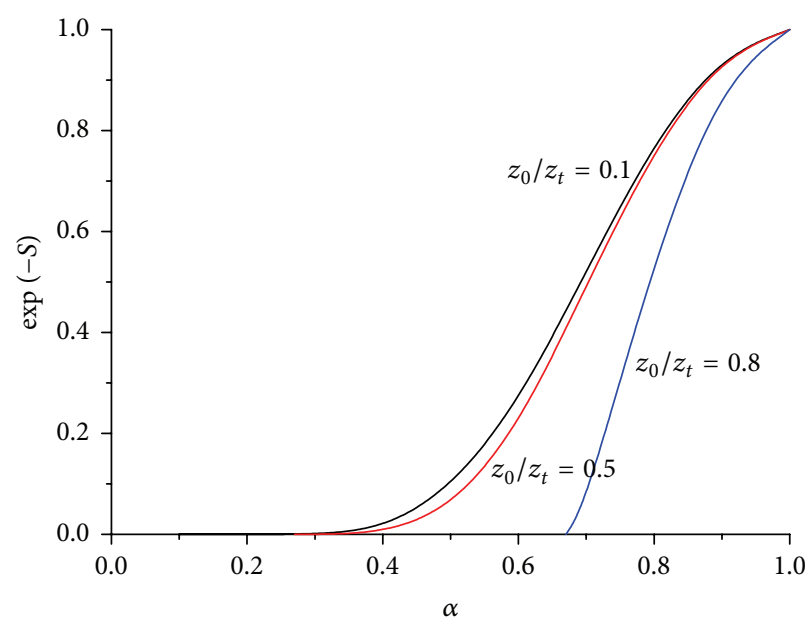

(b)

Figure 3: $S$ and $e^{-S}$ versus $\alpha$ with fixed $Q=0.2$. In all plots from left to right $z_{0} / z_{t}=0.1,0.5,0.8$.

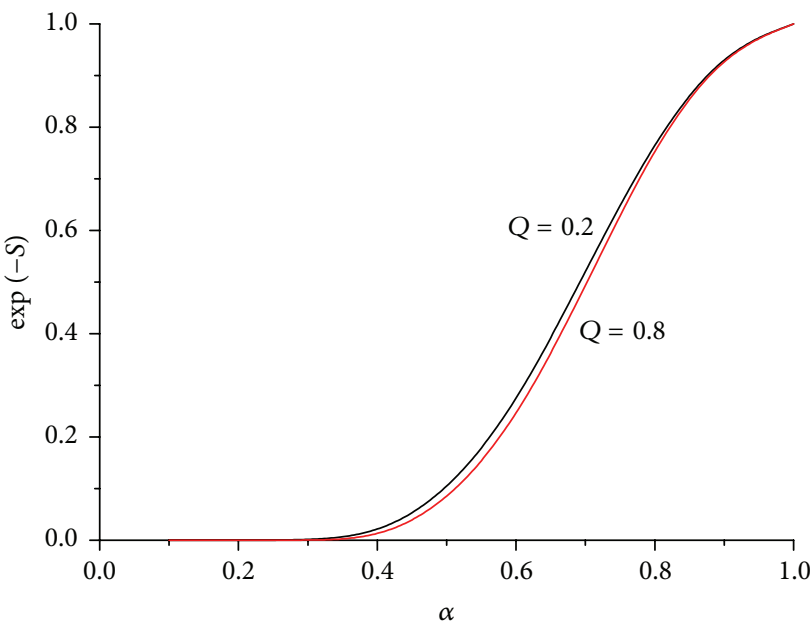

(a)

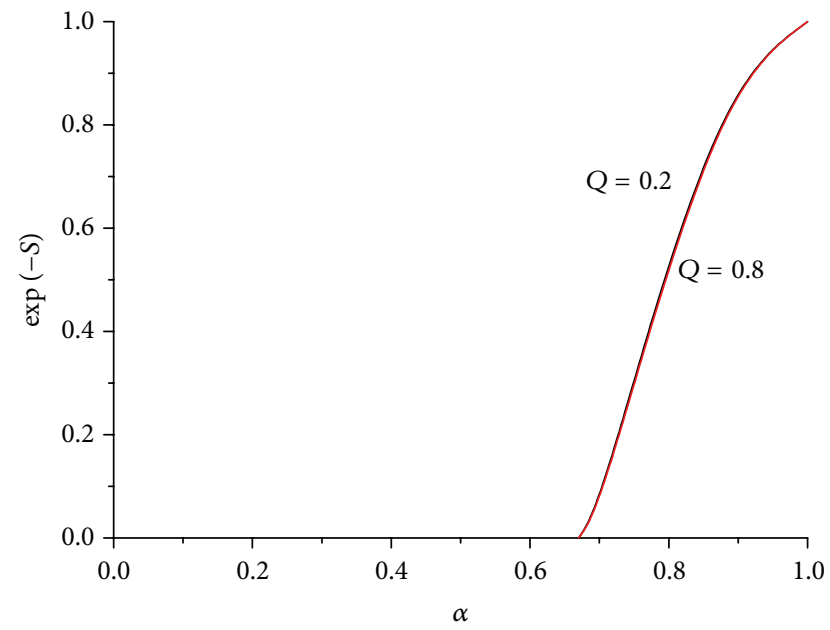

(b)

FIGURE $4: e^{-S}$ versus $\alpha$ with fixed $z_{0} / z_{t}$. (a) $z_{0} / z_{t}=0.1$. (b) $z_{0} / z_{t}=0.8$. In all plots from top to bottom $Q=0.2,0.8$.

the presence of strong external fields. In this paper, we have studied the effect of chemical potential on the holographic Schwinger effect by considering a confining D3-brane background with charge. The potential of a test particle pair was obtained by calculating the Nambu-Goto action of string attaching the rectangular Wilson loop at the probe D3-brane. The production rate for various cases was evaluated numerically. The effect of the chemical potential on the production rate was qualitatively shown. From the results, we can indeed see that there exist two critical values of the electric field. In addition, both the potential analysis and the numerical results of the production rate suggest that the presence of chemical potential tend to suppress the Schwinger effect. Interestingly, the instanton effects on the Schwinger effect have been studied in [30]. It is found that the presence of instantons also suppresses the pair production effect at finite temperature.

\section{Competing Interests}

The authors declare that they have no competing interests.

\section{Acknowledgments}

This research is partly supported by the Ministry of Science and Technology of China (MSTC) under the 973 Project no. 2015CB856904(4). Zi-qiang Zhang is supported by the NSFC under Grant no. 11547204. Gang Chen is supported by the NSFC under Grant no. 11475149. De-fu Hou is partly supported by NSFC under Grants nos. 11375070 and 11221504.

\section{References}

[1] J. S. Schwinger, "On gauge invariance and vacuum polarization," Physical Review Letters, vol. 82, no. 5, pp. 664-679, 1951. 
[2] I. K. Affleck and N. S. Manton, "Monopole pair production in a magnetic field," Nuclear Physics, Section B, vol. 194, no. 1, pp. 38-64, 1982.

[3] J. Maldacena, "The large $N$ limit of superconformal field theories and supergravity," Advances in Theoretical and Mathematical Physics, vol. 2, no. 2, pp. 231-252, 1998.

[4] S. S. Gubser, I. R. Klebanov, and A. M. Polyakov, "Gauge theory correlators from non-critical string theory," Physics Letters. B, vol. 428, no. 1-2, pp. 105-114, 1998.

[5] O. Aharony, S. S. Gubser, J. Maldacena, H. Ooguri, and Y. $\mathrm{Oz}$, "Large $N$ field theories, string theory and gravity," Physics Reports. A Review Section of Physics Letters, vol. 323, no. 3-4, pp. 183-386, 2000.

[6] A. S. Gorsky, K. A. Saraikin, and K. G. Selivanov, "Schwinger type processes via branes and their gravity duals," Nuclear Physics. B, vol. 628, no. 1-2, pp. 270-294, 2002.

[7] G. W. Semenoff and K. Zarembo, "Holographic Schwinger effect," Physical Review Letters, vol. 107, no. 17, Article ID 171601, 2011.

[8] Y. Sato and K. Yoshida, "Universal aspects of holographic Schwinger effect in general backgrounds," Journal of High Energy Physics, vol. 2013, no. 12, article 51, pp. 1-15, 2013.

[9] Y. Sato and K. Yoshida, "Holographic Schwinger effect in confining phase," Journal of High Energy Physics, vol. 2013, article 134, 2013.

[10] D. Kawai, Y. Sato, and K. Yoshida, "Schwinger pair production rate in confining theories via holography," Physical Review D, vol. 89, no. 10, Article ID 101901, 2014.

[11] Y. Sato and K. Yoshida, "Potential analysis in holographic Schwinger effect," Journal of High Energy Physics, vol. 2013, article 2, 2013.

[12] S. Chakrabortty and B. Sathiapalan, "Schwinger effect and negative differential conductivity in holographic models," Nuclear Physics. B, vol. 890, pp. 241-262, 2015.

[13] S. Bolognesi, F. Kiefer, and E. Rabinovici, "Comments on critical electric and magnetic fields from holography," Journal of High Energy Physics, vol. 2013, no. 1, article 174, 2013.

[14] Y. Sato and K. Yoshida, "Holographic description of the Schwinger effect in electric and magnetic field," Journal of High Energy Physics, vol. 2013, article 111, 2013.

[15] K. B. Fadafan and F. Saiedi, "On holographic non-relativistic Schwinger effect," http://arxiv.org/abs/1504.02432.

[16] S.-J. Zhang and E. Abdalla, "Gauss-Bonnet corrections to holographic Schwinger effect in confining back-ground," General Relativity and Gravitation, vol. 48, article 60, 2016.

[17] K. Hashimoto, T. Oka, and A. Sonoda, "Electromagnetic instability in holographic QCD," Journal of High Energy Physics, vol. 2015, article 1, 2015.

[18] J. Sadeghi, S. Tahery, and F. Razavi, "Schwinger effect and potential analysis in holographic QCD with deformation parameter," http://arxiv.org/abs/1603.07629.

[19] J. Ambjørn and Y. Makeenko, "Remarks on holographic Wilson loops and the Schwinger effect," Physical Review D, vol. 85, no. 6, Article ID 061901, 2012.

[20] K. Hashimoto and T. Oka, "Vacuum instability in electric fields via AdS/CFT: Euler-Heisenberg Lagrangian and Planckian thermalization," Journal of High Energy Physics, vol. 2013, no. 10, article 116, 2013.

[21] D. D. Dietrich, "Worldline holographic Schwinger effect," Physical Review D, vol. 90, no. 4, Article ID 045024, 11 pages, 2014.
[22] W. Fischler, P. H. Nguyen, J. F. Pedraza, and W. Tangarife, "Holographic Schwinger effect in de Sitter space," Physical Review D, vol. 91, no. 8, Article ID 086015, 2015.

[23] M. Ghodrati, "Schwinger effect and entanglement entropy in confining geometries," Physical Review D. Particles, Fields, Gravitation, and Cosmology, vol. 92, no. 6, Article ID 065015, 2015.

[24] X. Wu, "Notes on holographic Schwinger effect," Journal of High Energy Physics, vol. 2015, no. 9, article 44, pp. 1-12, 2015.

[25] D. Kawai, Y. Sato, and K. Yoshida, "A holographic description of the Schwinger effect in a confining gauge theory," International Journal of Modern Physics A, vol. 30, no. 11, Article ID 1530026, 2015.

[26] C. Ewerz, L. Lin, A. Samberg, and K. Schade, "Holography for heavy quarks and mesons at finite chemical potential," http://arxiv.org/abs/1511.04006.

[27] M. Cvetic and S. S. Gubser, "Phases of $R$-charged black holes, spinning branes and strongly coupled gauge theories," The Journal of High Energy Physics, vol. 1999, article 4, 1999.

[28] A. Chamblin, R. Emparan, C. V. Johnson, and R. C. Myers, "Charged AdS black holes and catastrophic holography," Physical Review D, vol. 60, no. 6, Article ID 064018, 1999.

[29] B. Zwiebach, A First Course in String Theory, Cambridge University Press, Cambridge, UK, 2004.

[30] M. Dehghani and P. Dehghani, "Holographic Schwinger effect in a D-Instanton background," https://arxiv.org/abs/1511.07986. 

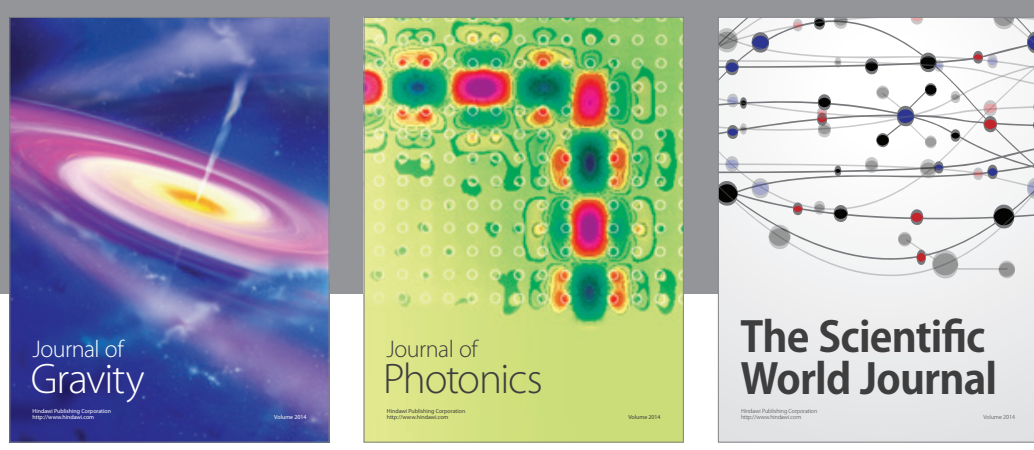

The Scientific World Journal
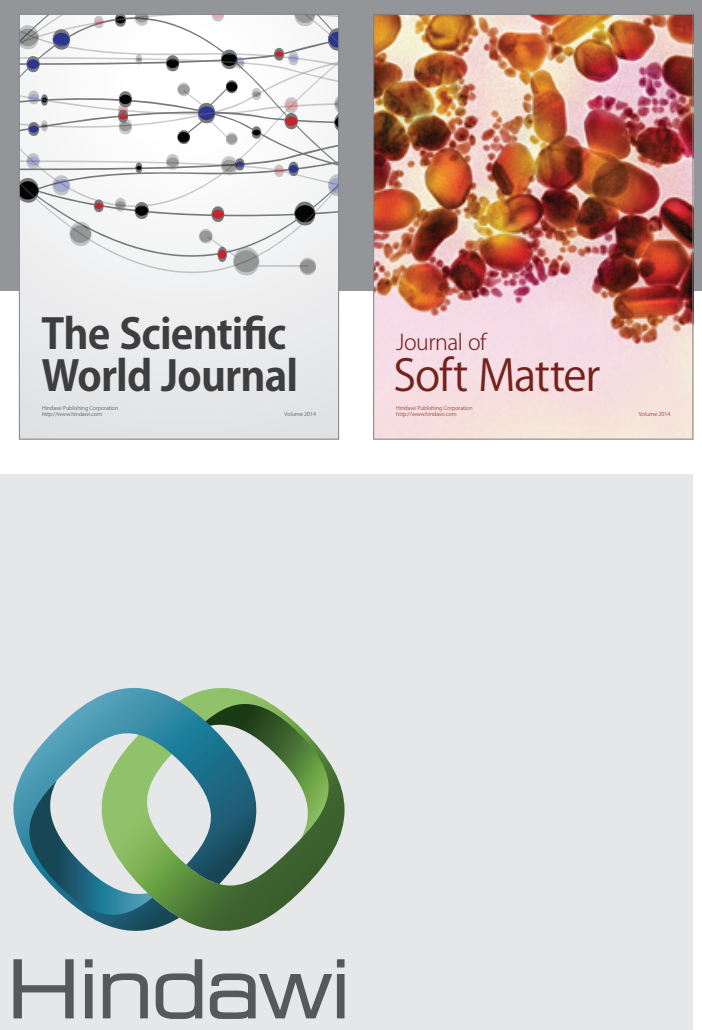

Submit your manuscripts at

http://www.hindawi.com

nternational Journal of

Statistical Mechanics
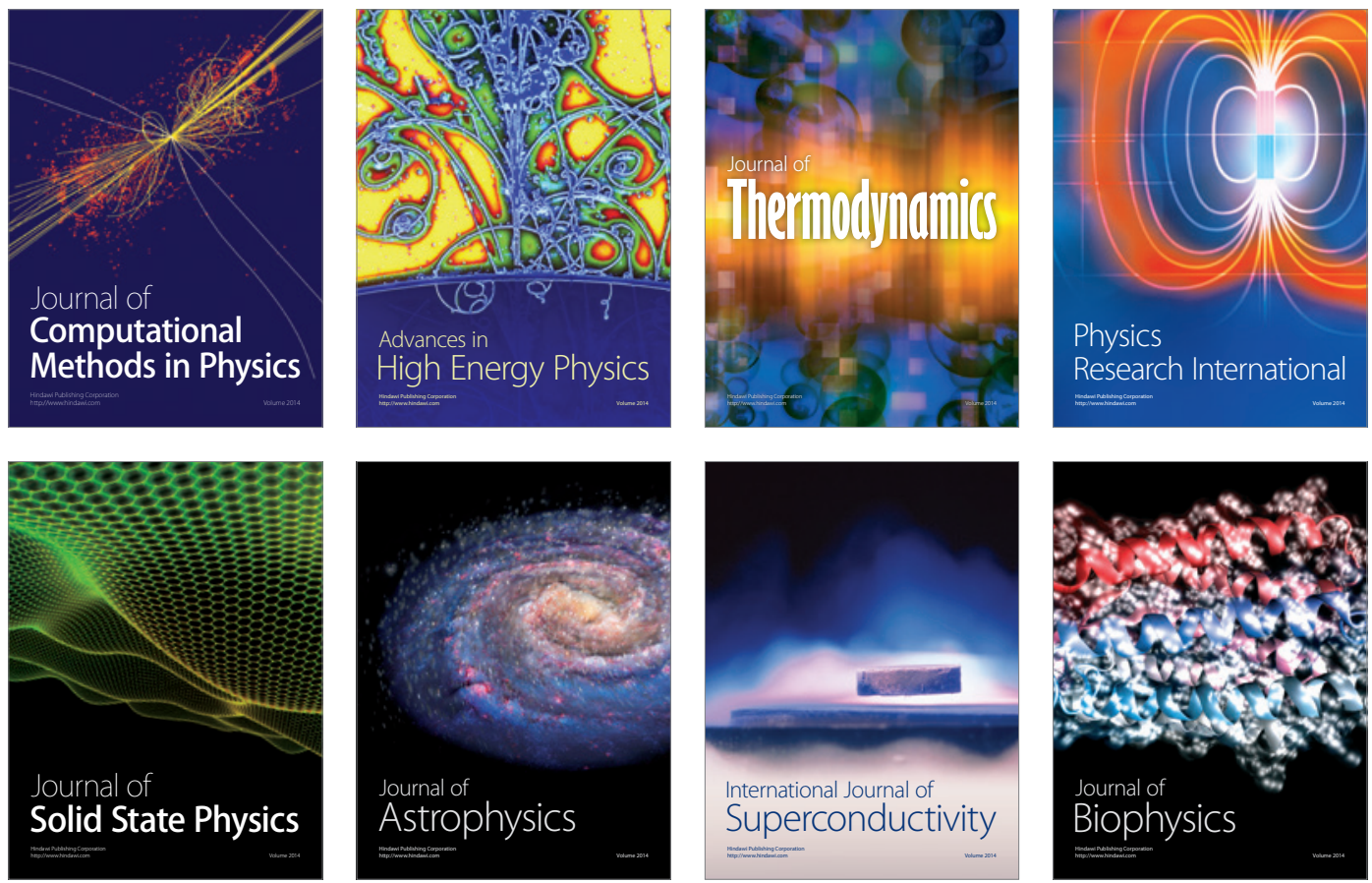
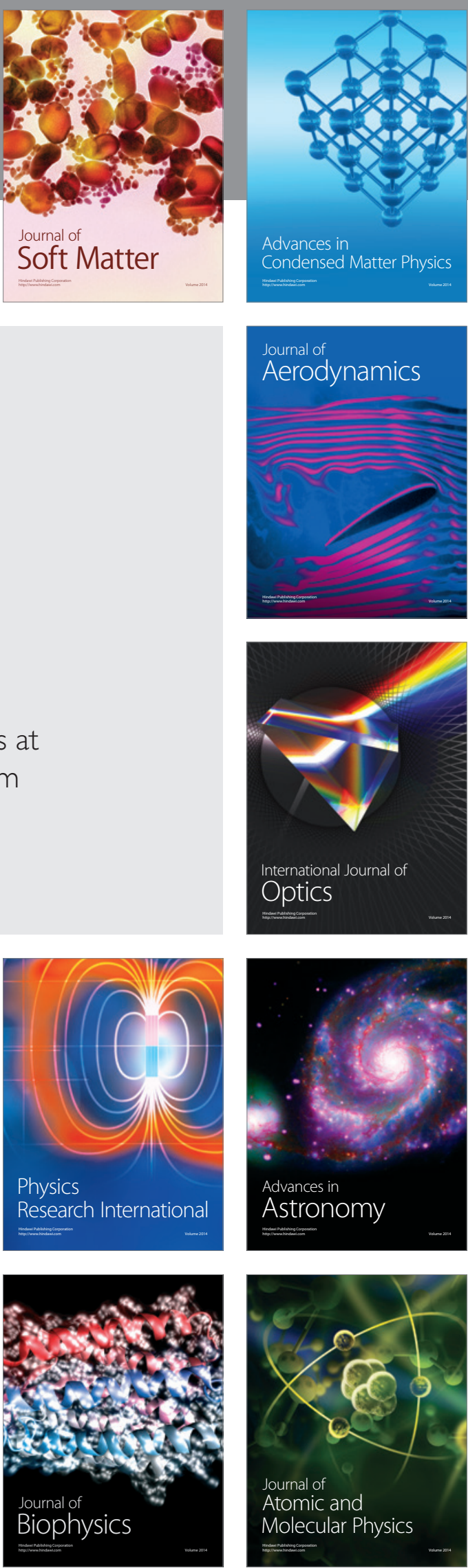